\title{
Entrenamiento Conductual y Atención Plena sobre el Estrés Parental y Relación Padre-Hijo
}

\author{
Yors Alexander Garcia ${ }^{1}$ \\ Fundacion Universitaria Konrad Lorenz \\ The Chicago School of Professional Psychology \\ Ivonne Andrea Grau \\ Fundacion Universitaria Konrad Lorenz
}

\begin{abstract}
RESUMEN - El objetivo principal de este estudio fue evaluar la efectividad de un procedimiento tradicional a padres combinado con uno de atención plena en la reducción del estrés parental e incremento en interacción positiva padre-hijo. Se utilizó un diseño pre test - pos test de un solo grupo. Durante el pre test y pos test se aplicaron el PSI, FFMQ y PCRI-M para evaluar el estrés parental, atención plena y relación padre-hijo, respectivamente. La intervención duró ocho semanas en que se implementó un procedimiento de entrenamiento de padres y uno de atención plena. Los resultados muestran que el entrenamiento combinado fue efectivo en la reducción del estrés parental, el incremento del uso de la atención plena y la mejora en la relación padre-hijo.
\end{abstract}

Palabras-Clave: estrés parental, atención plena, relación padre-hijo, conductas problema

\section{Treinamento Comportamental e de Mindfulness sobre o Estresse Parental e a Relação entre Pais e Filhos}

\begin{abstract}
RESUMO - O principal objetivo deste estudo foi avaliar a efetividade de um tradicional procedimento de treinamento de pais combinado a um treinamento de mindfulness na redução do estresse parental e no aumento de interações positivas entre país e filhos. Utilizou-se um delineamento com pré-teste e pós-teste e com um grupo. Durante as fases de pré-teste e pós-teste, aplicaram-se o PSI, o FFMO e o PCRI-M para avaliar estresse parental, mindfulness e relação entre país e filhos, respectivamente. A intervenção durou oito semanas, em que se implementaram o treinamento de pais e o de mindfulness. Os resultados demonstraram que o treinamento combinado foi efetivo na redução do estresse parental, no aumento de mindfulness e na melhora da relação etre país e filhos.
\end{abstract}

Palavras-Chave: estresse parental, mindfulness, relações país-criança, problemas comportamentais

\section{Behavioral Training and Mindfulness on Parental Stress and Parent-Child Relationship}

\begin{abstract}
The main objective of this study was to evaluate the effectiveness of a traditional parent training procedure combined with one of mindfulness in reducing parental stress and increasing positive parent-child interaction. A pretest-posttest design with one group was used. During the pretest and posttest phases the PSI, FFMQ and PCRI-M were applied to assess parental stress, mindfulness and parent-child relationship, respectively. The intervention lasted eight weeks in which a parent training procedure with mindfulness training was implemented. The results show that the combined training was effective in reducing parental stress, increasing mindfulness and improving parent-child relationship.
\end{abstract}

Keywords: parental stress, mindfulness, parent-child relationship, behavior problems

El modelo de intervención de entrenamiento conductual a padres considera que las prácticas parentales inefectivas evidencian la carencia de habilidades básicas para el manejo de conducta de los hijos, igualmente establece que parte de los factores mantenedores de conductas problema son las prácticas de crianza inconsistentes enmarcadas en contingencias de reforzamiento negativo en la interacción padre-hijo (Patterson, 2016). El curso repetitivo y escalonado en el que la conducta del padre es reforzada negativamente por la conducta del niño y viceversa, es conocido como el

1 Endereço para correspondência: Fundación Universitaria Konrad Lorenz, Carrera 9 $9^{\mathrm{a}}, \mathrm{n}^{\circ}$. 62-27, Bogotá, Colombia.E-mail: yorsgarcia@, yahoo.com ciclo coercitivo, que a largo plazo tiene efectos negativos para el desarrollo ajustado de la conducta infantil. Además, restringe las oportunidades para que el niño aprenda un espectro amplio de repertorios necesarios para la socialización, adaptación y autonomía (Dishion \& Snyder, 2016). De otra parte, la investigación en estrés parental ha demostrado que las estrategias asociadas al ciclo coercitivo, reducen temporalmente tanto los comportamientos problemáticos del niño, como el malestar emocional que experimenta el padre en estos contextos (Deater-Deckard, 1998; Kazdin \& Whitley, 2003). Finalmente, cabe mencionar que el establecimiento de una relación padre-hijo basada en una emocionalidad negativa, traza un factor de riesgo para 
el desarrollo de competencias de socialización y regulación emocional (Havighurst et al., 2015).

Investigadores como Anthony et al. (2005) resaltan que el estrés parental tiene un efecto directo sobre el desarrollo infantil, a la vez que afecta directamente la adquisición de competencias sociales y de regulación emocional que contribuirán al ajuste social del niño. Las reacciones emocionales negativas de los padres al estrés, y las respuestas no contingentes a las expresiones y necesidades emocionales del niño, fomentan contextos que le dificultan aprender estrategias adecuadas de regulación emocional y respuesta social efectiva, haciéndole más vulnerable al desarrollo de conductas problema (Kiel \& Kalomiris, 2015). Adicionalmente, la investigación ha mostrado que existen otros factores que contribuyen a una crianza negativa (e.g., ambigüedad en el establecimiento de límites y consecuencias, disciplina inconsistente, demandas desproporcionadas para la edad) (Berg-Nielsen, Vikan, \& Dahl, 2002; Chamberlain, Reid, Ray, Capaldi, \& Fisher, 1997; Crnic, Gaze, \& Hoffman, 2005). En general, la calidad de las interacciones padres-hijos como contexto precursor de los primeros sistemas de conducta, se ve afectada por las estrategias de afrontamiento, usualmente bajo condiciones de estrés, que los padres usan para responder a momentos de conflicto y atender contingentemente a las expresiones emocionales y necesidades del niño (Deater-Deckard, 1998; García, Cristiano, \& Grau, 2016; Hall \& Cook, 2011; Kiel \& Kalomiris, 2015).

Uno de los protocolos de intervención a padres basado en los principios operantes que ha recibido validez empírica en el trabajo con padres es el de McMahon y Forehand (2003), Ayudando al Niño Desobediente (Helping the Noncompliant Child [HNC, por sus siglas en inglés]; ver Kaminski, Valle, Filene, \& Boyle, 2008; McMahon \& Kotler, 2008, para una revisión de la evidencia empírica). Este protocolo fue diseñado específicamente para intervenir niños entre los 3 y los 8 años que presentan desobediencia persistente a las figuras adultas, considerando esta conducta como un fuerte predictor de problemas de comportamiento externalizante (Havighusrt et al., 2015; McMahon \& Kotler, 2008), déficit de atención e hiperactividad (Campbell, Shaw, \& Gilliom, 2000) y otras conductas disruptivas (Dishion \& Snyder, 2016).

Pese a la evidente efectividad de los programas de entrenamiento conductual a padres, estos modelos de entrenamiento presentan algunas limitaciones, por ejemplo, los procedimientos no están siempre adaptados a la población objetivo, los cambios en las conductas de los niños no son duraderas, y no intervienen aspectos emocionales de los niños (e.g., validación) (Kaminski et al., 2008; Lundahl, Risser, \& Lovejoy, 2006), ni de los padres (Shea \& Coyne, 2011). Una de las estrategias de intervención a padres que ha surgido en años recientes y que ha demostrado tener un impacto directo en la probabilidad de adherencia al tratamiento son las basados en atención plena, compasión y aceptación (Bögels, Hellemans, van Deursen, Römer, \& Meulen, 2014; Cairncross \& Miller, 2016; Dumas, 2005; Duncan, Coatsworth, \& Greenberg, 2009). Estos protocolos han mostrado beneficios clínicos al ser aplicables en distintos contextos y problemáticas conductuales, particularmente dentro del contexto de la interacción padre-hijo (Bögels \& Restifo, 2014; Kabat-Zinn \& Kabat-Zinn, 1998; Hall \& Cook, 2011). La habilidad de atención plena que se define como "prestar atención de manera intencional al momento presente, sin juzgar" (Kabat-Zinn \& Kabat-Zinn, 1998), ha servido de herramienta terapéutica para intervenir en procesos clínicos, como el estrés parental (Bögels et al., 2014), mejora de la relación padre-hijo (Brown, Whittingham, Boyd, McKinlay, \& Sofronoff, 2014; Coatsworth et al., 2015), decremento de la evitación experiencial en padres (Shea \& Coyne, 2011), aumento en conductas de cuidado, empatía y compasión (Dumas, 2005), y ha mostrado igualmente efectos positivos en la reducción de conductas externalizantes (e.g., agresión, desobediencia, inatención), e internalizantes en niños (e.g., depresión, ansiedad) (Singh et al., 2006; Van der Oord, Bögels, \& Peijnenburg, 2012).

Algunos investigadores (Bögels \& Restifo, 2014; Dumas, 2005; Singh et al., 2006) argumentan que uno de los mecanismos de cambio que contribuye a la mejora de las interacciones padres-hijos es el decremento de los procesos automáticos de crianza; es decir, la rigidez con la que se aplican las diferentes prácticas de crianza. Por ejemplo, los padres utilizan las mismas estrategias de crianza (discutir, amenazar o golpear) ante diferentes problemas de conducta (desobediencia, agresión), sin tomar en consideración aspectos funcionales de la conducta y emoción del niño. Por tal razón, alguno autores como Bögels y Restifo, (2014), Cohen y Semple (2010), Dumas (2005), Duncan et al. (2009) han venido investigando una manera más eficaz de romper el ciclo de rigidez comportamental de las prácticas de crianza, esta consiste en utilizar estrategias de atención plena. Varios de estos estudios han mostrado la necesidad de integrar procesos como la atención plena, aceptación, compasión, valores, entre otros, junto con los procedimientos tradicionales de entrenamiento conductual a padres. Esta tarea de integración transparadigmática no representa un eclecticismo conceptual, sino más bien, el desarrollo de un trabajo teórico coherente de intervención clínica en padres. Recientemente varios investigadores han venido integrando conceptual y metodológicamente estos dos modelos de intervención con resultados clínicamente significativos (Brown et al., 2014, Coatsworth et al., 2015; Cohen \& Semple, 2010; Duncombe et al., 2016; Sanders, Kirby, Tellegen, \& Day, 2014; Singh et al., 2006; Van der Oord et al., 2012; Whittingham, 2014).

Teniendo en cuenta la efectividad que tienen las estrategias de atención plena en la mejora de las prácticas de crianza, decremento de estrés parental, mejora en la relación padre-hijo, se hace necesario realizar más estudios empíricos que validen la incorporación de estas estrategias dentro de los modelos operantes de intervención a padres. La razón de utilizar tanto las estrategias tradicionales de control operante (reforzamiento diferencial, extinción, moldeamiento, etc.), como las de atención plena, radica en el hecho que variables inherentes al proceso de crianza como regulación emocional, validación, y aceptación de eventos privados negativos, no son intervenidas directamente por las estrategias operantes clásicas de intervención a padres (ver Cohen \& Semple, 2010; Whittingham, 2014). El efecto positivo de esta alianza presenta un desafío conceptual, metodológico y aplicado 
en esta área de intervención. Por ejemplo, algunos autores (Dumas, 2005; Singh et al., 2006; Whittingham, 2014) han iniciado programas de intervención que buscan avanzar en la validación de estos protocolos de intervención, sin embargo, estos aun no logran integrar completamente cada uno de los procesos utilizados en ambos modelos. Con base en lo anterior, se hace necesario incrementar la validez empírica de la aplicación de estos dos paradigmas de intervención en el entrenamiento a padres. En esta línea, el presente trabajo intenta evaluar la relevancia de combinar estas dos estrategias de entrenamiento a padres, entrenamiento tradicional y atención plena, e identificar si tiene un efecto significativo en la reducción de conductas parentales que están relacionadas con los procesos de crianza, en este caso particular, el estrés parental y el incremento de las interacciones positivas en la relación padre-hijo.

Como se describió anteriormente, uno de los protocolos con mayor soporte empírico en el trabajo con padres es el HNC (McMahon \& Forehand, 2003), el cual está exclusivamente basado en el entrenamiento de las estrategias operantes a padres (extinción, atención), sin embargo, recientemente algunos autores (ver Parent et al., 2014; Parent, McKee, Rough, \& Forehand, 2016), han evaluado el papel de estrategias de atención plena dentro de este procedimiento y su relación con el proceso de crianza (e.g., relación padrehijo, estrategias de control parental). Aunque los resultados son prometedores, los estudios son pocos, por lo tanto se requiere mayor trabajo en área para evaluar la validez de este protocolo combinado con atención plena en variables centrales del proceso de crianza como es el estrés parental y la calidad de la relación padre-hijo. Por otra parte, uno de los protocolos en atención plena para padres que más ha recibido validez empírica es el de Bögels y Restifo, (2014). Por lo tanto, este estudio busca validar la efectividad de ambos protocolos en el decremento de estrés parental e incremento en la relación positiva padre-hijo.

\section{Método}

\section{Participantes}

En esta investigación participaron un total de cinco madres, dos padres de familia y seis niños, sin embargo es importante mencionar que los niños no fueron parte directa del proceso de entrenamiento. Esta decisión se tomó con base en aspectos logísticos, y debido a que el protocolo de atención plena requería la asistencia única de los padres. En la Tabla 1 se presentan las características sociodemográficas de todos los participantes. Inicialmente un total de 18 madres y padres fueron remitidos directamente a los investigadores principales por parte de las directivas de un jardín infantil. Los padres fueron remitidos a los investigadores por presentar dificultades en prácticas de crianza, y los niños problemas de conducta. Luego de este proceso, el número de padres se redujo a siete a través del cumplimiento de los criterios de inclusión como fueron puntajes altos en las pruebas de Estrés Parental (PSI), y puntajes bajos en el cuestionario de Cinco Facetas de Atención Plena (FFMQ), y cuestionario de
Tabla 1. Características Sociodemográficas de Padres y Niños

\begin{tabular}{lcc}
\hline & Participantes $(\mathrm{N}-7)$ & Niños $(\mathrm{N}=6)$ \\
\hline Promedio Edad & 33,57 & 4,57 \\
Total Genero & & 7 \\
Feminino & 5 & 0 \\
Masculino & 2 & \\
Grado escolar hijo & & 3 \\
Pre Jardín & N/A & 3 \\
Transición & N/A & 1 \\
Parvulo & N/A & \\
Ocupación Actual & & N/A \\
Empleado & 6 & N/A \\
Hogar & 1 & \\
Estado Civil & & N/A \\
Casado & 4 & N/A \\
Unión de hecho & 3 & N/A \\
Nivel de Estudios & 4 & \\
Bachillerato & & \\
Técnico & & \\
Universitario & & \\
\hline
\end{tabular}

Crianza Parental versión para madres (PCRI-M). Finalmente, es importante mencionar que este estudio fue avalado por el comité de ética de la universidad que financió el estudio (\# 201301-004). Igualmente, este cumplió con las normas éticas establecidas en el código deontológico Colombiano. Cada uno de los padres firmó un consentimiento informado.

\section{Diseño}

Para esta investigación se utilizó un diseño pre test-post test de un solo grupo (Kazdin, 2003). Específicamente, luego que los padres fueron remitidos por la institución colaboradora se procedió a la aplicación de los siguientes tres cuestionarios (PSI, FFMQ, y PCRI-M). Una vez los padres con puntajes clínicamente significativos en el PSI, y bajos en el FFMQ y PCRI-M fueron seleccionados, recibieron ocho sesiones de entrenamiento semanal de 2 horas y media en ambos protocolos. Durante este período se entrenó a los participantes en habilidades parentales utilizando el protocolo de McMahon y Forehand, (2003), y el de atención plena para padres (Bögels \& Restifo, 2014). Una vez terminado este proceso se aplicaron nuevamente los tres instrumentos junto a un cuestionario de satisfacción.

\section{Lugar, Instrumentos y Materiales}

La investigación se llevó a cabo en la institución que remitió a los padres para que hicieran parte de este entrenamiento. Las sesiones de entrenamiento se realizaron semanalmente en un salón de clase, el cual contenía sillas, colchonetas, un computador, un proyector y material didáctico como lápices, tijeras, papel y cubos. Igualmente se hizo entrega a los padres de folders donde podían archivar 
semanalmente copias de cada una de las guías de trabajo y hojas de registro.

Índice de Estrés Parental - Versión Corta. El Índice de Estrés Parental- Versión Corta (Parental Stress Index-Short Form [PSI]) es un cuestionario de 36 preguntas que mide el nivel de estrés generado por el rol parental a través de tres dimensiones (malestar paterno, interacción disfuncional padres-hijos, y estrés relacionado con la dificultad del niño). Puntajes superiores al percentil 85 son indicadores de niveles clínicos de estrés. La prueba se responde en una escala tipo Likert (que tan acuerdo están hasta fuertemente en desacuerdo), donde los puntajes altos indican altos niveles de estrés y los bajos respuesta defensiva. La escala muestra un test-retest para un período de 6 meses de (.78.85) y una confiabilidad interna (.80-.87) (Díaz-Herrero et al. 2011). Para este estudio se utilizó una versión validada de esta prueba al español, la cual ha demostrado tener una consistencia interna para las sub-escalas de malestar paterno y niño difícil (.90 y .87, respectivamente) (Díaz-Herrero et al. 2011).

Cuestionario de las Cinco Facetas de Atención Plena. El cuestionario de Cinco Facetas de Atención Plena (Five Facets Mindfulness Questionnaire [FFMQ]) es un cuestionario de 39 preguntas que mide la atención plena diaria a través de cinco factores (observar, describir, actuar dándose cuenta, no juzgar la experiencia interna, y no reactividad sobre la experiencia interna). La prueba se responde en una escala tipo Likert donde se indica el nivel de acuerdo $(1=$ "nunca o muy raramente verdad" hasta $5=$ "muy a menudo o siempre verdad") (Baer, Smith, Hopkins, Krietemeyer, \& Toney, 2006). Puntajes altos en la suma total de las cinco sub escalas indica un alto uso de la atención plena durante el día. Para este estudio se utilizó la versión en español del FFMQ (Cebolla et al., 2012). Esta versión muestra una alta consistencia interna (sub escalas $\alpha=0.80$ a 0.91 ).

Cuestionario de Crianza Parental. El cuestionario de Crianza Parental versión para madres (Parent-Child Relationship Inventory [PCRI-M]) es un cuestionario de 78 preguntas que mide las actitudes de los padres hacia la crianza de los hijos a través de ocho escalas (apoyo parental, satisfacción con la crianza, compromiso, comunicación, disciplina, autonomía, distribución de rol, y deseabilidad social). La prueba se responde en una escala tipo Likert en la que se indica el nivel de acuerdo (1= "muy de acuerdo" hasta 5= "en total desacuerdo") (Gerard, 1994). En general, puntajes altos en cualquiera de las escalas indica una buena actitud hacia la crianza, mientras que puntajes bajos indica prácticas de crianza poco efectivas. Para esta investigación se utilizó la adaptación del PCRI-M a población española (Roa $\&$ del Barrio, 2001). Se debe de mencionar que la adaptación española excluye las escala de disciplina, por tal esta razón solo se evaluaron siete escalas. La versión española presenta favorable validez de constructo.

Cuestionario de Satisfacción. Al final del estudio se administró un cuestionario de satisfacción especialmente diseñado para evaluar la validez social de estos programas (McMahon \& Forehand, 2003). El cuestionario cuenta con 47 preguntas en total, distribuidas en varias dimensiones (programa general, formato de enseñanza, técnicas específicas de crianza, ejecución del terapeuta durante el proceso de entrenamiento y opiniones generales sobre cambios realizados, progreso, resultados y manejo de las conductas problema de los niños). Las preguntas se responden en una escala Likert $(1=$ "considerablemente peor" hasta $7=$ "considerablemente mejor").

\section{Procedimiento}

A continuación se describen las diferentes fases por las que pasaron cada uno de los padres participantes de este estudio.

Pretest. En esta fase se procedió a administrar uno de los cuestionarios de evaluación funcional de la conducta (O’Neill, Albin, Storey, Horner, \& Sprague, 2014) para establecer tanto las topografías como la función de las conductas disruptivas que presentaban los niños en casa. Igualmente se administraron las pruebas del PSI, FFMQ y el PCRI-M, y los padres firmaron el consentimiento informado.

Entrenamiento conductual a padres. Para este módulo se utilizó el modelo de entrenamiento a padres de McMahon y Forehand, (2003). Este programa está dividido principalmente en el aprendizaje de dos grandes paquetes de habilidades, una es atención diferencial en la cual los padres aprenden a observar y reforzar diferencialmente conductas apropiadas, mientras ignoran las conductas inapropiadas. En el segundo módulo los padres aprenden formas más efectivas de dar instrucciones a sus hijos y asegurarse que estos las sigan. El protocolo está diseñado para padres, y niños entre los 3-8 años que presentan conductas disruptivas en casa. Para el entrenamiento se utilizó un protocolo de entrenamiento en habilidades comportamentales (ver Miltenberger, 2016). Es decir, primero se explicó la habilidad, seguido por el modelamiento de la misma por parte de los dos investigadores, luego se realizó un juego de roles utilizando un análisis de tareas, y finalmente se dio retroalimentación a los padres sobre la ejecución de la misma. En esta investigación solo se utilizó el protocolo de entrenamiento con padres debido a los objetivos particulares del estudio, los niños no hicieron parte directa del entrenamiento. Igualmente se utilizaron otras estrategias de evaluación, en este caso una funcional, que difiere del protocolo original. Finalmente el entrenamiento se realizó en grupo, a diferencia del protocolo original. Es importante mencionar que ninguno de estos cambios afecta significativamente la utilidad del protocolo (Long \& Forehand, 2000). A continuación se describen brevemente las dos principales fases que comprenden este protocolo de entrenamiento a padres.

Fase 1. Habilidades de atención diferencial. Antes de iniciar con el entrenamiento formal de las habilidades en esta fase, se procedió a realizar en una sesión aparte la introducción de los objetivos del protocolo, número de sesiones, formato de entrenamiento y compromisos de los padres durante el proceso. El objetivo de ésta fase fue entrenar a los padres a que discriminaran cómo funciona el ciclo coercitivo de interacciones negativas entre padres e hijos. En la segunda reunión se inició con la primera sesión formal de entrenamiento en la cual a los padres se les enseñó a prestar más atención a las conductas apropiadas de los niños, y reducir el número de instrucciones, preguntas y 
críticas durante las interacciones diarias. En la tercera sesión se entrenó a los padres a reforzar correctamente, verbal y físicamente, la ejecución de conductas apropiadas de los niños, e ignoraran las inapropiadas. En la cuarta sesión se procedió a entrenar el uso de la extinción a través de ignorar conductas inapropiadas y, en la quinta y última sesión de esta fase se entrenó a los padres a que utilizaran de forma efectiva la atención diferencial, la cual consistía en el uso combinado de reforzamiento diferencial y extinción (ignorar). En total esta fase duró cinco semanas de entrenamiento.

Fase 2. Entrenamiento en obediencia. El objetivo de esta fase fue entrenar a los padres a dar instrucciones claras y apropiadas a los niños. Específicamente, los padres fueron entrenados a dar instrucciones correctamente a través de la implementación de un protocolo de tres fases. En la primera fase instruccional (opción A), los padres aprendieron a dar instrucciones claras, una a la vez, cortas y concisas; el objetivo fue que aprendieran a dar la instrucción y esperar a que el niño la pudiera seguir por lo menos dentro de los primeros 5 seg. La segunda fase instruccional (opción B) consistía en dar advertencias apropiadamente a los hijos si estos no cumplían con lo demandado en la instrucción dentro de los primeros $5 \mathrm{seg}$. (eg. "si no haces...te voy a poner en tiempo fuera o con la variante de tiempo para pensar en cómo hacerlo mejor"). En ambas fases se le pidió a los padres que atendieran y reforzaran específicamente el cumplimiento de la instrucción. En la última fase de seguimiento instruccional (opción C), los padres fueron entrenados a utilizar el tiempo fuera efectivamente si los niños no cumplían con lo requerido en la instrucción. Se hizo especial énfasis en un uso positivo del tiempo fuera, es decir, se entrenó a los padres maximizar la calidad, cantidad y duración del reforzamiento positivo dentro de las actividades diarias, así como el uso del tiempo fuera con y sin exclusión (ver Miltenberger, 2016 para más detalles). Finalmente, en la última sesión de esta fase se entrenó a los padres en la implementación de las instrucciones de forma más flexible y generalizada por el seguimiento y cumplimiento de las mismas.

Entrenamiento en atención plena a padres. El protocolo entrenamiento en atención plena a padres de Bögels y Restifo (2014) se utilizó en este estudio. El objetivo de este programa fue entrenar a los padres para utilizar las estrategias de atención plena durante el proceso de crianza, a reaccionar diferencialmente ante el estrés, y desarrollar conductas de autocuidado, empatía y compasión hacia ellos mismos y sus hijos. El protocolo está dividido en 8 sesiones, las cuales se describen más adelante. Se utilizó el mismo procedimiento de entrenamiento en habilidades conductuales descrito anteriormente. Es decir, primero se describía la habilidad a entrenar, posteriormente se practicaba junto con los padres, y finalmente se daba retroalimentación por la ejecución realizada. Adicionalmente los padres registraron diariamente la práctica de cada una de las habilidades de atención plena. A diferencia del protocolo original, y debido al objetivo de la investigación, la mitad de la sesión (1 hora y media) se utilizó para entrenar estos módulos. A continuación se describen brevemente las ochos diferentes sesiones.

Sesión 1. Paternidad automática. El objetivo fue entrenar a los padres en discriminar respuestas automáticas (e.g., en la programación diaria de actividades) en situaciones de estrés con sus hijos. Específicamente, se entrenó a los padres para actuar con mayor atención a las reacciones internas de malestar y señales de estrés que produce la crianza de los hijos.

Sesión 2. Mente parental de principiante. El objetivo de esta sesión fue entrenar a los padres en observar a los niños desde una perspectiva diferente, es decir que aprendieran a observar a los niños sin preconcepciones (e.g., inatento, insoportable, irrespetuoso, etc.). Igualmente se entrenó a los padres en estrategias de auto-compasión.

Sesión 3. Reconectándose con su cuerpo como padre. El objetivo de esta sesión fue entrenar a los padres para explorar de formas más directas las reacciones físicas que se presentan bajo estrés. Igualmente se entrenó a los padres en reconocer las señales físicas de incomodidad que se ignoran por estar bajo el control directo de las necesidades diarias de los niños.

Sesión 4. Respondiendo vs reaccionando al estrés parental. En esta fase se entrenó a los padres en discriminar las diferentes reacciones físicas que produce el estrés, y a estar en mayor contacto con ellas. En esta fase se entrenó a los padres para explorar las diferentes reacciones fisiológicas del estrés, y cómo responder de forma consciente a ellas.

Sesión 5. Patrones de crianza y esquemas. En esta fase los padres fueron entrenados en identificar las prácticas de crianza bajo las cuales fueron educados, y a discriminar si esas eran las mismas prácticas que utilizaban con sus hijos. Igualmente, en esta fase se entrenó a los padres en discriminar las prácticas de crianza punitivas que usan con sus hijos. Finalmente se enseñó a los padres a utilizar otras estrategias de auto compasión.

Sesión 6. Conflicto y crianza. En esta sesión los padres fueron entrenados para discriminar los conflictos que surgen en la relación padre-hijo, y a partir de ellos crear nuevos patrones de interacción positiva. En esta fase se continuó con el entrenamiento en estrategias de compasión hacia ellos mismos y sus hijos.

Sesión 7. Amor y límites. En esta sesión se entrenó a los padres para practicar el autocuidado ante situaciones emocionales intensas y negativas. Específicamente, se entrenó a los padres a que discriminaran cuando sus hijos transgredían sus límites personales, y a proceder con estrategias de reparación. Finalmente, se entrenó a los padres para establecer de forma clara y adecuada un ambiente más estructurado para sus hijos.

Sesión 8. Un camino de atención plena a través de la crianza. En la última sesión se revisaron las diferentes dificultades que tuvieron los padres para llevar a la práctica cada una de las habilidades entrenadas.

Post test. El post test fue exactamente similar al pre test, con la excepción que junto a los tres cuestionarios (PSI, FFMQ y PCRI-M) se administró el cuestionario de satisfacción. En esta última sesión se realizó el cierre y retroalimentación con los padres de las dificultades encontradas en la aplicación de ambos protocolos y habilidades aprendidas.

\section{Resultados}

Las medias y desviaciones estándar de cada uno de los puntajes del pre test y post test de los tres instrumentos 
Tabla 2. Medias y Desviaciones Estándar del Índice de Estrés Parental, FFMQ y PCRI-M

\begin{tabular}{|c|c|c|c|c|}
\hline & \multicolumn{2}{|c|}{ Pre test } & \multicolumn{2}{|c|}{ Post Test } \\
\hline & Media & $\mathrm{DE}$ & Media & $\mathrm{DE}$ \\
\hline \multicolumn{5}{|l|}{ Estrés Parental - PSI } \\
\hline Malestar Paterno & 33,29 & 9,99 & 24,29 & 8,38 \\
\hline Interacción. Padre-Hijo & 28,57 & 7,20 & 19,29 & 6,89 \\
\hline Niño Difícil & 34,71 & 8,05 & 23,86 & 6,28 \\
\hline Puntaje Total & 96,57 & 20,66 & 67,43 & 17,33 \\
\hline \multicolumn{5}{|l|}{ FFMQ } \\
\hline Observar & 19,86 & 4,74 & 23,14 & 2,54 \\
\hline Describir & 29 & 8,04 & 30,14 & 7,58 \\
\hline Actuar dándose cuenta & 25 & 5,16 & 27,43 & 5,79 \\
\hline No Juzgar experiencia interna & 25,29 & 5,70 & 28,29 & 5,55 \\
\hline No reactivida experi. interna & 20,43 & 3,59 & 22,43 & 2,37 \\
\hline Puntaje Total & 119,58 & 27,25 & 131,4 & 23,85 \\
\hline \multicolumn{5}{|l|}{ PCRI-M } \\
\hline Apoyo parental & 22,57 & 3,86 & 26,86 & 6,14 \\
\hline Satisfacción crinaza & 33,43 & 4,46 & 33,14 & 10,4 \\
\hline Compromiso & 43,86 & 4,92 & 44,57 & 8,20 \\
\hline Comunicación & 26,29 & 3,25 & 27,86 & 6,54 \\
\hline Autonomía & 25 & 5,71 & 26,57 & 3,55 \\
\hline Distribución de rol & 25,71 & 3,09 & 28 & 7,16 \\
\hline Deseabilidad social & 14,86 & 1,95 & 15,86 & 3,76 \\
\hline
\end{tabular}

(PSI, FFMQ y PCRI-M) se presentan en la Tabla 2. Para evaluar las diferencias estadísticas entre las medidas pre test y post test de los tres instrumentos se aplicó una prueba $t$ de muestras dependientes. Los datos presentaron distribución normal. Los resultados muestran que existió una diferencia estadísticamente significante en la medida de estrés total $\mathrm{t}(6)=7.61, \mathrm{p}<.000$, es decir, los puntajes de estrés total decrecieron significativamente en el post test comparado con la medida inicial de pre test. Con respecto a los tres factores del PSI (malestar paterno, interacción disfuncional padre-hijo y niño difícil), los resultados muestran que los puntajes decrecieron significativamente en el post test. Específicamente, se encontró una diferencia estadísticamente significativa en el decremento del malestar paterno $t(6)=$ $4.30, p<.005$, interacción disfuncional padre-hijo $t(6)=$ $4.49, p<.004$, y el factor de niño difícil $t(6)=7.63, p<$ .000. En general, con respecto al Índice de Estrés Parental, los resultados muestran que la intervención combinada de entrenamiento tradicional a padres y atención plena fue efectiva en el decremento del estrés parental.

Por otra parte, los resultados del instrumento de las Cinco Facetas de Atención Plena (FFMQ) muestra que existió una diferencia estadísticamente significativa en el incremento de la práctica de atención plena en los participantes $t(6)=$ $-4.583, p<.004$. Con respecto a cada uno de los factores de la atención plena que mide el instrumento, los resultados evidencian una diferencia estadísticamente significativa en el factor de observación $t(6)=-2.860, p<.029$, descripción $t(6)=-3.361, \mathrm{p}<.015$, actuar dándose cuenta $t(6)=-3.740$, $p<.010$, no juzgar la experiencia interna $t(6)=-3.813, p$ $<.009$, y no reactividad sobre la experiencia interna $t(6)=$
$-2.449, p<.050$. En general, los resultados muestran que la intervención en atención plena a los padres participantes fue efectiva en la práctica de la misma durante el ejercicio diario del rol parental. Finalmente, con respecto al cuestionario de crianza parental (PCRI-M) los resultados del puntaje total de las ocho escalas muestra que la interacción entre padres e hijos incrementó significativamente $t(6)=-2.871, p<$ .028. Sin embargo, con respecto a cada una de las escalas los puntajes muestran algunas diferencias importantes. Específicamente, los datos muestran que existió una diferencia estadísticamente significativa en las escala de apoyo parental $t(6)=-3.116, p<.021$, escala de satisfacción con la crianza $t(6)=-2.328, p<.059$, escala de compromiso $\mathrm{t}(6)=-4.600, \mathrm{p}<.004$, y escala de disciplina $t(6)=-3.750$, $p<.010$. Por otra parte, en la escala de comunicación $t(6)=$ $-.990, p=.360$, escala de autonomía $t(6)=-.894, \mathrm{p}=.406$, escala de atribución del rol $t(6)=-.739, p=.488$, y escala de deseabilidad social $t(6)=-.734, p=.491$ no mostraron diferencias estadísticamente significativas. En general, los resultados muestran que la interacción padre-hijo presentó incremento significativamente solamente en las escalas de apoyo parental, satisfacción con la crianza, compromiso y disciplina, mientras que en las otras escalas no se presentó diferencia alguna. Sin embargo el puntaje global del PCRI-M muestra un incremento significativo en la relación padre-hijo.

Los datos de la escala de satisfacción aplicada a los padres al final del programa de intervención se analizaron a través de una estadística descriptiva. La escala toma puntajes de 1 a 7 donde $(1=$ "considerablemente peor" hasta $7=$ “considerablemente mejor"). Específicamente, los datos muestran que los padres reportaron que el entrenamiento en atención plena y habilidades de manejo contingencial mejoró las conductas problema de sus hijos, y la interacción con ellos en la casa $(M=5.35, D E=0.475)$. Con respecto al formato de enseñanza del programa, los padres encontraron fácil $(M=2.27, D E=1.03)$ y de gran utilidad $(M=6.41$, $D E=0.42$ ) realizar cada una de las actividades entrenadas. Respecto a las técnicas específicas de crianza, los padres encontraron una moderada dificultad en la aplicación de estas $(M=3.47, D E=0.372)$, igualmente reportaron que fue de gran utilidad el uso de la atención plena y manejo contingencial en la mejora de las prácticas de crianza $(M=$ $6.38, D E=0.418$ ). En general, los datos del cuestionario de satisfacción muestran que los padres encontraron de gran utilidad la aplicación de las estrategias de crianza, y que estas tuvieron un efecto significativo en la disminución de las conductas problemas en casa.

\section{Discusión}

En general, los resultados del estudio muestran que tras el procedimiento combinado de entrenamiento conductual a padres y atención plena, los puntajes en estrés parental decrecieron significativamente, mientras que los puntajes en la relación padre-hijo incrementaron en comparación al pre test. Estos resultados confirman la efectividad de los procedimientos conductuales de entrenamiento a padres (Cairncross \& Miller, 2016; Kaminski et al., 2008; Lundahl et al., 2006), y de los procedimientos que utilizan estrategias de 
atención plena (Van der Oord et al., 2012) en la reducción del estrés parental y la mejora en la relación padre-hijo (Duncan et al., 2009). Sin embargo, es importante resaltar nuevamente, que este es uno de los pocos estudios que combinan ambos procedimientos para decrecer el estrés parental y mejorar la relación padre-hijo (ver también, Singh et al., 2006). Por otra parte, este estudio valida igualmente los hallazgos de otros estudios que han mostrado un efecto significativo en el uso de estrategias de atención plena y mejora en la relación padre-hijo (Bögels et al., 2014; Brown et al., 2014; Dumas, 2005; Parent et al., 2016). En conclusión, los resultados de este estudio muestran un efecto significativo de las estrategias combinadas de entrenamiento a padres y atención plena sobre las variables de interés como fueron el estrés parental, atención plena y relación padre-hijo. Finalmente, aunque esta investigación utilizó un formato grupal, los resultados son igualmente efectivos como cuando se utilizan individualmente (Bögels \& Restifo, 2014; McMahon \& Forehand, 2003).

Aunque el procedimiento combinado de entrenamiento a padres en general fue efectivo, es importante revisar en detalle el impacto que tuvo el entrenamiento sobre cada una de las variables de estudio. Específicamente, los puntajes en estrés presentaron decrementos significativos en los tres factores del índice de estrés parental (PSI) (malestar paterno, interacción disfuncional padre-hijo y niño difícil) tras la intervención. Los resultados de esta investigación validan los resultados de estudios similares (Deater-Deckard, 1998; Kazdin \& Whitley, 2003). Por otra parte, con respecto a los factores del cuestionario de cinco facetas de atención plena (FFMQ; observar, describir, actuar dándose cuenta, no juzgar la experiencia interna, y no reactividad sobre la experiencia interna), los resultados muestran un incremento significativo en los puntajes tras el entrenamiento de esta habilidad. Sin embargo, es importante mencionar que el objetivo de este estudio fue evaluar si el entrenamiento en atención plena, no solo incrementaba su práctica, sino el efecto que podría tener sobre la reducción en el estrés parental y la mejora en la calidad de la relación padrehijo. Con respecto al decremento en estrés parental, los resultados confirman que fue efectivo, sin embargo con respecto a algunos componentes de la relación padre-hijos los resultados no fueron totalmente exitosos. Particularmente los resultados muestran que en el cuestionario de crianza parental (PCRI-M) las únicas escalas que no mostraron significancia estadística fueron: comunicación, autonomía, atribución del rol y deseabilidad social. Al respecto surgen algunas hipótesis de estos resultados. Primero, a excepción de dos participantes, el resto de ellos incluyó solo a un miembro de la pareja durante las sesiones de entrenamiento. La evidencia muestra que es una variable que afecta significativamente la efectividad en la aplicación de los procedimientos operantes, particularmente en lo que tiene que ver con la aplicación contingente de las consecuencias (Bagner \& Eyberg, 2003). Variables como la autonomía y atribución de rol se ven seriamente afectadas por estos factores dado que solo un padre es quien ejerce la función de control sobre las conductas de los hijos. Segundo, el formato de entrenamiento fue con los padres exclusivamente, y como muestra la investigación (Kamisnki et al., 2008), los procedimientos que incluyen a los niños en el entrenamiento son más efectivos, especialmente en variables como la comunicación. En conclusión, los resultados muestran que aunque el procedimiento combinado fue efectivo, algunas variables directamente relacionadas a la conformación de la familia y el módulo de entrenamiento afectaron algunos de los factores que miden la relación padre-hijo.

La combinación de las estrategias de atención plena y control contingencial han mostrado ser eficaces tanto en el manejo de conductas problemáticas en niños, como en la reducción del estrés parental. A nivel clínico estas estrategias presentan resultados prometedores en la medida que proveen de herramientas al psicólogo infantil en áreas como la aceptación y validación de emociones en niños y padres, regulación emocional más eficaces a largo plazo, compasión y auto-compasión de los padres hacia el proceso de crianza, clarificación de valores y atención plena (Brown et al., 2015; Coatsworth et al., 2015; Kabat-Zinn \& KabatZinn, 1998). Todas estas estrategias han mostrado un efecto significativo en variables relacionadas con el proceso de crianza como son el estrés parental, conductas problema, desobediencia, ansiedad infantil, entre otras. Por tal razón es de gran importancia incorporar de forma coherente dentro del marco de los procedimientos operantes este tipo de estrategias de intervención en la práctica clínica con padres. Por ejemplo, algunos estudios recientes han empezado a incorporar en el entrenamiento a padres estrategias como la regulación emocional (Ben-Porath, 2010, Ducombe et al., 2016; Havighusrt et al., 2015) y auto-compasión (Neff $\&$ Faso, 2014) dentro de sus modelos de intervención. Se requiere más investigación en área, y que los modelos de control contingencial hagan uso de estas herramientas para intervenir en variables que no han formado tradicionalmente parte de sus objetivos terapéuticos (ver Smith \& Dishion, 2013, para un ejemplo).

Algunas limitaciones se pueden mencionar en este estudio. Primero, solo se contó con un grupo experimental, lo que puede limitar en parte el alcance de los resultados. Segundo, el formato de entrenamiento fue grupal, el protocolo de McMahon y Forehand, (2003) está diseñado para trabajo individual, aunque se han hecho algunos estudios bajo el formato grupal (McMahon \& Kotler, 2008). En parte el formato grupal pudo limitar el nivel de manejo y aprendizaje de las habilidades entrenadas en los padres. Tercero, aunque los padres puntuaron alto en el post test del cuestionario de atención plena, esta habilidad no se pudo medir directamente en la interacción con los padres, lo que potencialmente limita la aplicación de la habilidad en la interacción padre-hijo. Cuarto, la exclusión directa de los niños de las fases de entrenamiento de habilidades y de las mediciones directas pudo ser un factor directo en los pocos cambios que se presentaron en áreas como la comunicación en el PCRI-M, esto es un factor directo de cambios en la relación padre-hijo en este tipo de protocolos (Eyberg \& Bussing, 2010). Finalmente, una limitación metodológica que ha sido mencionada en otros estudios, tiene que ver con las dificultades de los diseños para identificar y abstraer conclusiones sobre potenciales mecanismos de cambio (Kaminski et al., 2008). 
Por otra parte, son varias las alternativas de futuras investigaciones que pueden salir a partir de este estudio. La más importante es evaluar por separado los componentes de atención plena y protocolo tradicional con el objetivo de validar cuál de los dos tiene mayor efecto en la reducción del estrés parental y mejora de la relación padre-hijo. Igualmente, es importante validar el protocolo en el decremento conductas internalizantes (ansiedad) en niños. Además es importante evaluar la efectividad del protocolo con padres de niños mayores, para determinar la variabilidad que surge como resultado del proceso de crianza en momentos evolutivos diferenciales o para encontrar la generalización del modelo. Finalmente, se sugiere en investigaciones futuras, evaluar el efecto del protocolo en poblaciones con mayor vulnerabilidad, por ejemplo, cuidadores de niños con discapacidades en el desarrollo y otras discapacidades, teniendo en cuenta que la literatura indica que el bienestar psicológico y físico de estos padres sufre más efectos negativos a causa del estrés en la crianza y cuidado que requieren los niños con desarrollo típico y más aún si presentan alteraciones en el desarrollo (Whittingham, 2014; (Whittingham, Sanders, McKinlay \& Boyd, 2015).

En conclusión, este estudio evidenció la relevancia empírica y clínica que tiene el uso de estrategias de atención plena y control contingencial en el decremento de estrés parental y mejora de la relación padre-hijo.

\section{Referencias}

Abidin, R.R. (1995). Parenting Stress Index (PSI) manual (3rd ed.). Charlottesville, VA: Pediatric Psychology Press.

Anthony, L. G., Anthony, B. J., Glanville, D. N., Naiman, D. Q., Waanders, C., \& Shaffer, S. (2005). The relationships between parenting stress, parenting behaviour and preschoolers' social competence and behaviour problems in the classroom. Infant and Child Development, 14, 133-154.

Baer, R. A., Smith, G. T., Hopkins, J., Krietemeyer, J., \& Toney, L. (2006). Using self-report assessment methods to explore facets of mindfulness. Assessment, 13, 27-45.

Bagner, D. M., \& Eyberg, S. M. (2003). Father involvement in parent training: When does it matter? Journal of Clinical Child and Adolescent Psychology, 32, 599-605.

Ben-Porath, D. D. (2010). Dialectical behavior therapy applied to parent skills training: Adjunctive treatment for parents with difficulties in affect regulation. Cognitive and Behavioral Practice, 17, 458-465.

Berg-Nielsen, T. S., Vikan, A., \& Dahl, A. A. (2002). Parenting related to child and parental psychopathology: A descriptive review of the literature. Clinical Child Psychology and Psychiatry, 7, 529-552.

Bögels, S., \& Restifo, K. (2014). Mindful parenting: A guide for mental health practitioners. New York, NY: Springer.

Bögels, S. M., Hellemans, J., van Deursen, S., Römer, M., \& van der Meulen, R. (2014). Mindful parenting in mental health care Effects on parental and child psychopathology, parental stress, parenting, coparenting, and marital functioning. Mindfulness, $5,536-551$
Brown, F. L., Whittingham, K., Boyd, R. N., McKinlay, L., \& Sofronoff, K. (2014). Improving child and parenting outcomes following paediatric acquired brain injury: A randomised controlled trial of stepping stones triple P plus acceptance and commitment therapy. Journal of Child Psychology and Psychiatry, 55, 1172-1183.

Cairncross, M., \& Miller, C. J. (2016). The effectiveness of mindfulness-based therapies for ADHD: A meta-analytic review. Journal of Attention Disorders, 20, 1-17.

Campbell, S. B., Shaw, D. S., \& Gilliom, M. (2000). Early externalizing behavior problems: Toddlers and preschoolers at risk for later maladjustment. Development and Psychopathology, $12,467-488$

Cebolla, A., Garcia-Palacios, A., Soler, J., Guillen, V., Baños, R., \& Botella, C. (2012). Psychometric properties of the Spanish validation of the five facets of mindfulness questionnaire (FFMQ). The European Journal of Psychiatry, 26, 118-126.

Chamberlain, P., Reid, J. B., Ray, J., Capaldi, D. M., \& Fisher, P. (1997). Parent Inadequate Discipline (PID). In T. A. Widiger, A. J. Frances, H. A. Pincus, R. Ross, M. B. First, \& W. Davis (Eds.), DSM-IV sourcebook (Vol. 3, pp. 569-629). Washington, DC: American Psychiatric Association.

Crnic, K. A., Gaze, C., \& Hoffman, C. (2005). Cumulative parenting stress across the preschool period: Relations to maternal parenting and child behaviour at age 5. Infant and Child Development, 14, 117-132.

Coatsworth, J. D., Duncan, L. G., Nix, R. L., Greenberg, M. T., Gayles, J. G., ... Demi, M. A. (2015). Integrating mindfulness with parent training: Effects of the mindfulness-enhanced strengthening families program. Developmental Psychology, $51,26-35$

Cohen, J. A. S., \& Semple, R. J. (2010). Mindful parenting: A call for research. Journal of Child and Family Studies, 19, 145-151.

Deater-Deckard, K. (1998). Parenting stress and child adjustment: Some old hypotheses and new questions. Clinical Psychology: Science and Practice, 5, 314-332.

Díaz-Herrero, Á., López-Pina, J. A., Pérez-López, J., de la Nuez, A. G. B., \& Martínez-Fuentes, M. T. (2011). Validity of the parenting stress index-short form in a sample of Spanish fathers. The Spanish Journal of Psychology, 14, 990-997.

Dishion, T.J., \& Snyder, J. J. (2016). The Oxford handbook of coercive relationship dynamics. New York: Oxford University Press.

Dumas, J. E. (2005). Mindfulness-based parent training: Strategies to lessen the grip of automaticity in families with disruptive children. Journal of Clinical Child and Adolescent Psychology, 34, 779-791.

Duncan, L. G., Coatsworth J. D., \& Greenberg, M. T. (2009). A model of mindful parenting: Implications for parent-child relationships and prevention research. Clinical Child Family Psychology, 12, 255-270.

Duncombe, M. E., Havighurst, S. S., Kehoe, C. E., Holland, K. A., Frankling, E. J., \& Stargatt, R. (2016). Comparing an emotion-and a behavior-focused parenting program as part of a multsystemic intervention for child conduct problems. Journal of Clinical Child \& Adolescent Psychology, 45(3), 320-334. 
Eyberg, S. M., \& Bussing, R. (2010). Parent-child interaction therapy for preschool children with conduct problems. In R. C. Murrihy, A. D. Kidman \& T. H. Ollendick (Eds.), Clinical handbook of assessing and treating conduct problems in youth (pp. 139-162). New York: Springer Science.

Gerard, A. (1994). Parent-child relationship inventory: Manual. Los Angeles: Westem Psychological Services.

Hall, K., \& Cook, M. (2011). The power of validation: Arming your child against bullying, peer pressure, addiction, self-harm, and out-of-control emotions. New Harbinger Publications.

Havighurst, S. S., Duncombe, M., Frankling, E., Holland, K., Kehoe, C., \& Stargatt, R. (2015). An emotion-focused early intervention for children with emerging conduct problems. Journal of Abnormal Child Psychology, 43(4), 749-760.

Kabat-Zinn, M., \& Kabat-Zinn, J. (1998). Everyday blessings: The inner work of mindful parenting. Hyperion.

Kaminski, J. W., Valle, L. A., Filene, J. H., \& Boyle, C. L. (2008). A meta-analytic review of components associated with parent training program effectiveness. Journal of Abnormal Child Psychology, 36, 567-589.

Kazdin, A. E. (2003). Research design in clinical psychology. Needham Heights, MA: Allyn \& Bacon.

Kazdin, A. E., \& Whitley, M. K. (2003). Treatment of parental stress to enhance therapeutic change among children referred for aggressive and antisocial behavior. Journal of Consulting and Clinical Psychology, 71, 504-515.

Kiel, E. J., \& Kalomiris, A. E. (2015). Current themes in understanding children's emotion regulation as developing from within the parent-child relationship. Current Opinion in Psychology, 3, 11-16.

Long, N., \& Forehand, R. (2000). Modifications of a parental training program for implementation beyond the clinical setting. In N. N. Singh, J. P. Leung, \& A. N. Singh (Eds.), International perspectives on child and adolescent mental health (pp. 293-310). New York: Elsevier.

Lundahl, B., Risser, H. J., \& Lovejoy, M. C. (2006). A meta-analysis of parent training: Moderators and follow-up effects. Clinical Psychology Review, 26, 86-104.

McMahon, R. J., \& Forehand, R. L. (2003). Helping the noncompliant child: Family-based treatment for oppositional behavior. New York: Guilford Press.

McMahon, R. J., \& Kotler, J. S. (2008). Evidence-based therapies for oppositional behavior in young children. In Handbook of evidence-based therapies for children and adolescents (pp. 221-240). New York: Springer.

Miltenberger, R. G. (2016). Behavior modification: Principles and procedures (6th ed.). Pacific Grove, CA: Wadsworth.

Neff, K. D., \& Faso, D. J. (2014). Self-compassion and well-being in parents of children with autism. Mindfulness, 12, 78-98
O’Neill, R. E., Albin, R. W., Storey, K., Horner, R. H., \& Sprague, J. R. (2014). Functional assessment and program development. Cengage Learning.

Parent, J., Clifton, J., Forehand, R., Golub, A., Reid, M., \& Pichler, E. R. (2014). Parental mindfulness and dyadic relationship quality in low-income cohabiting black stepfamilies: Associations with parenting experienced by adolescents. Couple and Family Psychology: Research and Practice, 3, 67-82

Parent, J., McKee, L. G., Rough, J. N., \& Forehand, R. (2016). The association of parent mindfulness with parenting and youth psychopathology across three developmental stages. Journal of Abnormal Child Psychology, 44, 191-202.

Patterson, G. R. (2016). Coercion theory: The study of change. In T. J. Dishion \& J. J. Snyder (Eds), The Oxford handbook of coercive relationship dynamics (pp. 7-22). New York: Oxford University Press.

Roa, L., \& del Barrio, M. V. (2001). Adaptación del Cuestionario de Crianza Parental (PCRI-M) a población española. Revista Latinoamericana de Psicología, 33, 329-341.

Sanders, M. R., Kirby, J. N., Tellegen, C. L., \& Day, J. J. (2014). The Triple P-Positive Parenting Program: A systematic review and meta-analysis of a multi-level system of parenting support. Clinical Psychology Review, 34(4), 337-357.

Shea, S. E., \& Coyne, L. W. (2011). Maternal dysphoric mood, stress, and parenting practices in mothers of Head Start preschoolers: The role of experiential avoidance. Child \& Family Behavior Therapy, 33, 231-247.

Singh, N. N., Lancioni, G. E., Winton, A. S., Fisher, B. C., Wahler, R. G., ... Sabaawi, M. (2006). Mindful parenting decreases aggression, noncompliance, and self-injury in children with autism. Journal of Emotional and Behavioral Disorders, 14, 169-177.

Smith, J. D., \& Dishion, T. J. (2013). Mindful parenting in the development and maintenance of youth psychopathology. In J. T. Ehrenreich-May \& B. C. Chu (Eds.), Transdiagnostic mechanisms and treatment for youth psychopathology (pp. 138-160). New York, NY: Guilford Press.

Van der Oord, S., Bögels, S. M., \& Peijnenburg, D. (2012). The effectiveness of mindfulness training for children with ADHD and mindful parenting for their parents. Journal of Child and Family Studies, 21, 139-147.

Whittingham, K. (2014). Parents of children with disabilities, mindfulness and acceptance: A review and a call for research. Mindfulness, 5, 704-709.

Whittingham, K., Sanders, M. R., McKinlay, L., \& Boyd, R. N. (2015). Parenting intervention combined with acceptance and commitment therapy: A trial with families of children with cerebral palsy. Journal of Pediatric Psychology, 41(5), 531-542. 